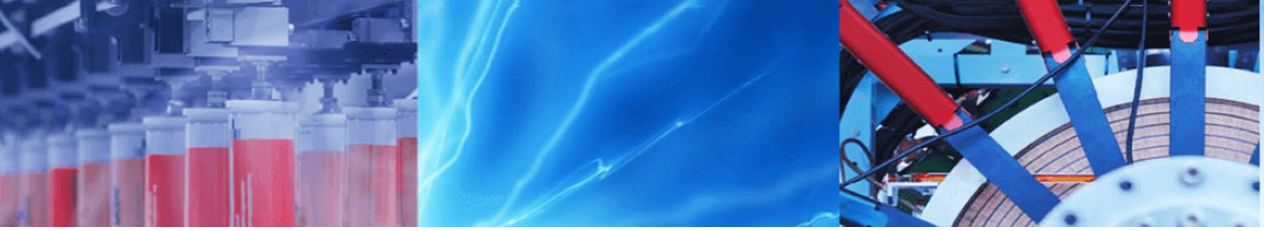

Research Article

\title{
A ternary system oleophilic-hydrophobic membrane prepared by electrospinning for efficient gravity-driven oil-water separation
}

\author{
Lu Wang $^{1} \cdot$ Shanshan Dai $^{1} \cdot$ Xuewei Liu $^{2} \cdot$ Xuerui Wang $^{3} \cdot$ Hongsheng Lu ${ }^{1}$ (D)
}

(c) Springer Nature Switzerland AG 2019

\begin{abstract}
The preparation of composite nanofibers often can make up for the shortage of single materials in the field of oil-water separation, including separation efficiency, mechanical properties and fine flexibility. The film materials prepared by doping inorganic nanoparticles have defects of poor stability and uneven dispersion of the fiber surface. In this paper, we prepared a novel polyvinylidene fluoride (PVDF) -polystyrene (PS) -cellulose acetate (CA) ternary mixture system membrane by electrospinning. Nanosized polystyrene in the spinning process uniformly gathers on the fiber surface by interlacing PVDF and CA to manipulate the microstructure of the fiber surface. PVDF is added to increase mechanical properties. Degradable CA is selected to promote pore formation and compatibility between the other two polymers. The composite membrane has high separation efficiency, high oil flux and superior mechanical properties as demonstrated by oil/water separation test and stress-strain test. The optimized oil flux reaches $1935.67 \mathrm{~L} \mathrm{~h}^{-1} \mathrm{~m}^{-2}$ only driven by gravity of $0.73 \mathrm{kPa}$. Due to low energy consumption, excellent separation and more flexible regulation, this membrane may have great application value in industrial oil-water separation in the future.
\end{abstract}

Keywords Electrospinning $\cdot$ Membrane $\cdot$ PVDF $\cdot$ Oil-water separation

\section{Introduction}

Oil spills have occurred frequently historically [1], From the Gulf of Mexico oil spill to the Bohai Bay [2-4], every accident has caused tremendous economic losses and environmental pollution, even threaten the entire marine life chain and human survival $[5,6]$. Therefore, the handling of oil spills is critical $[7,8]$. Traditional methods including gravity separation, slag removal, flotation, adsorption and biological treatment were used to deal with oil spills [4, 9-13]. However, the commonly used methods have their own limits such as low efficiency, incomplete separation, high cost and cumbersome process [1, 14].
In recent years, with the development of interface science and bionics, the researchers are eager to pursue materials that have opposite wettability to oil or water instead of traditional methods for oil-water separation [15]. The advantages of low cost and high separation efficiency make polymer membranes widely explored and applied $[2,16,17]$. Furthermore, it has great application prospects in industrial separation under various environments in handling marine leakage accidents $[18,19]$. The surface energy, specific surface area and the surface roughness are three crucial constituents for the separation efficiency of the membrane and the rate of oil-water separation [12]. Therefore, the choices of membrane substrate

Electronic supplementary material The online version of this article (https://doi.org/10.1007/s42452-019-0805-9) contains supplementary material, which is available to authorized users.

$\triangle$ Xuerui Wang, x.wang-12@tudelft.nl; $₫$ Hongsheng Lu, hshlu@163.com |'School of Chemistry and Chemical Engineering, Southwest Petroleum University, Chengdu 610500, People's Republic of China. ${ }^{2}$ Research Institute of Petroleum Engineering, PetroChina Dagang Oilfield Company, Tianjin 300280, People's Republic of China. ${ }^{3}$ Chemical Engineering Department, Delft University of Technology, Van der Maasweg 9, 2629 HZ Delft, The Netherlands. 
and the preparation process tempt the above three conditions to a tremendous extent.

The electrospinning process is simple in technology, low cost and can control the diameter of the fiber [20], from the nanometer to the micron level, the electrospun nanofiber material with a three-dimensional spatial structure not only has a small size and large specific surface area [21-23], but also has the characteristics of good mechanical stability, small pore size, high porosity and outstanding fiber continuity $[24,25]$. Therefore, it is an aggressive approach to prepare the oil-water separation membrane by electrospinning technology [26]. Previously, electrospun membrane materials have been widely reported for oil-water separation [27-29]. Polymers such as polyacryIonitrile [7], polyvinylidene fluoride [30], polyethersulfone [31], polystyrene [2], polylactic acid [32], polycaprolactone [21], etc. have been used for electrospinning. In addition, it is a universal research direction to combine surface modification technology of fibers or addition of inorganic nanoparticles with electrospinning [21]. Ahmed et al. mixed PVDF and hexafluoropropylene for spinning to increase mechanical strength [33]; Arslan et al. reported electrospun cellulose acetate modified by perfluoroethoxysilane. Arslan et al. [24] It has also been reported that inorganic nanoparticles were added to the spinning solution to increase mechanical strength. So for example, Obaid et al. [34] incorporated graphene oxide and silica nanoparticles into polysulfone; Liu et al. [32] reported that Ag nanoparticles were immobilized on polylactic acid fibers by dopamine. However, there are still many problems to be solved in the preparation of oil-water separation membranes by electrospinning. It is difficult for the membrane composed of a pure material to meet various requirements of oil-water separation simultaneously, including stability, strength, and separation efficiency [35]. These inorganic nanoparticles are easily detached from the fibers by this heavy oil and it is inhomogeneous and uncontrollable between the dispersion and agglomeration of the fiber surface $[16,27]$; Due to the precise fiber structure of electrospinning, there may be plugging pores or broken fiber structures by the viscosity and high density of the oil. We may be able to make further improvements in two ways. First, exploration of new spinnable materials, second, the preparation of multi-component composite fibers, including the compounding of between organic materials, the compounding of organic and inorganic materials and the compounding of between inorganic materials.

In this article, we fabricated a highly oleophilic and hydrophobic PVDF-PS-CA membrane that can separate oil-water mixtures only driven by gravity of $0.73 \mathrm{kPa}$. CA is considered to be a low-cost, green material with good biocompatibility and biodegradability and it is easy to form porous structure fibers and gain a high specific surface area [36]. PS is not directly involved in the formation of fibers, but as a "modified" material, forming very uniform organic nanoparticles about $10 \mathrm{~nm}$ on the surface of the fibers, increasing roughness [37]. The optimum ratio of PS and CA has an enormous impact on separation performance, so we explored the most suitable ratio. One limitation of the PS-CA membrane is the reduction in fiber strength and poor reusability. PVDF was added to improve the mechanical properties and reusability [19, 38,39 ]. Finally, we obtained oil-water separation membrane with high separation efficiency and high mechanical strength. Simultaneously, it has high oil flux only driven by gravity force. Undoubtedly this energy-saving and highefficiency separation material has promising potential for oil-water separation.

\section{Experimental}

\subsection{Materials}

Polystyrene (PS, Mw $=220,000 \mathrm{~g} / \mathrm{mol}$ ) and cellulose acetate $(\mathrm{CA}, \mathrm{Mw}=30,000 \mathrm{~g} / \mathrm{mol}$, acetyl $39.8 \mathrm{wt} \%$, hydroxyl $3.5 \mathrm{wt} \%)$ were purchased from Aladdin Industrial Corporation; Polyvinylidene fluoride (PVDF, $M w=1,200,000 \mathrm{~g} /$ mol) was purchased from Dongguan Zhanyang Polymer Materials Co. Ltd; N, N-dimethylformamide ( $\geq 99.5 \%)$ and chloroform $(\geq 99.0 \%)$ were purchased from Chengdu Cologne Chemical Co. Ltd. Sudan III and methylene blue also were purchased from Chengdu Cologne Chemical Co. Ltd. All chemicals were used as received without further purification.

\subsection{Preparation of PS-CA and PVDF-PS-CA membrane}

The PS-CA films were electrospun as follows: the concentration of the spinning solution is set to $15 \%$. Using DMF as a solvent for both polymers and the ratio of PS and CA was 3:7, 5:5 and 7:3 forming three different ratios of electrospun films named $\mathrm{M}-0-3 / 7, \mathrm{M}-0-5 / 5$ and $\mathrm{M}-0-7 / 3$. The details as follows: taking the membrane $\mathrm{M}-0-3 / 7$ as an example, $0.9 \mathrm{~g}$ of PS and $2.1 \mathrm{~g}$ of CA are dissolved in $17 \mathrm{~g}$ of DMF in a conical flask placed on a magnetic stirrer $12 \mathrm{~h}$ at $30^{\circ} \mathrm{C}$. Then take $5 \mathrm{~mL}$ of the spinning solution in a disposable needle tube and spray it with a 22-gauge needle. The needle tube was clamped on the booster. Mental needle was connected to high voltage generator. Distance between the spinneret and collector was set to $10 \mathrm{~cm}$. Then adjust the electrospinning positive and negative voltage to $10 \mathrm{kV}$ and $-5 \mathrm{kV}$ to get the most stable and uniform Taylor cone and set humidity to $40 \%$, temperature to $25^{\circ} \mathrm{C}$, spinning rate to $0.1 \mathrm{~mL} / \mathrm{h}$. Aluminum foil was cut 
into $10 \times 10 \mathrm{~cm}$ size and placed on the flat collector. After the spinning solution was completely sprayed, aluminum foil was taken out and dried in a vacuum oven at $60^{\circ} \mathrm{C}$ for $12 \mathrm{~h}$. The ratio of PS to CA was changed and membranes $\mathrm{M}-0-5 / 5, \mathrm{M}-0-7 / 3$ were prepared as above method.

Likewise, we set the total spinning solution concentration to $15 \%$ to prepare PVDF-PS-CA solution. The ratio of PS to CA is fixed at 3:7. PVDF is added and its concentration is adjusted to $2.5 \mathrm{wt} \%, 5 \mathrm{wt} \%, 7.5 \mathrm{wt} \%, 10 \mathrm{wt} \%, 12.5 \mathrm{wt} \%$, 15 wt\% getting 6 different ratios of membranes labeled as $\mathrm{M}-2.5-3 / 7, \mathrm{M}-5-3 / 7, \mathrm{M}-7.5-3 / 7, \mathrm{M}-10-3 / 7, \mathrm{M}-12.5-3 / 7$ and $M-15-0 / 0$. The preparation method of the spinning solution and the electrospinning process are consistent with the PS-CA system. At the same concentration, we prepared a PVDF: CA ratio of 2:1 as a contrast experiment without PS. The preparation process of membranes is shown in Fig. 1. Information on all membranes is summarized in Table 1.

\subsection{Separation performance test}

The oil absorption performance was measured using the ASTM F726-2012 sorbent sorption performance test method [40]. $10 \mathrm{~g}$ oil and $150 \mathrm{~mL}$ water were added to a $200 \mathrm{~mL}$ beaker, and then $0.05 \mathrm{~g}$ of the membranes was gently placed in the chloroform-water mixture. After $5 \mathrm{~min}$, the film was taken out with tweezers. The droplets suspended outside the adsorbent were air-dried. The adsorption capacity is the ratio of the mass of the adsorbed oil to the mass of the dry film. The formula is as follows:

$Q_{\text {oil }}=\frac{m_{0}-m_{1}}{m_{1}}$

$Q_{o i l}$ is the adsorption capacity $(\mathrm{g} / \mathrm{g})$ of the membrane, $m_{0}$ is the total mass $(\mathrm{g})$ of the wet membrane after the membrane is fully adsorbed, and $m_{1}$ is the mass $(\mathrm{g})$ of the dry membrane. The adsorption capacity of the nine different membranes was measured respectively.

The dried film was cut into a circular diaphragm with a diameter of $5 \mathrm{~cm}$ fixed in the middle of the separator. A measuring cylinder was used to weigh $75 \mathrm{~mL}$ chloroform and $75 \mathrm{~mL}$ water dyed with Sudan III and methylene blue respectively. The weighed chloroform and water were poured into a large beaker, $100 \mathrm{~mL}$ of the mixture was used as a separating solution, and another $50 \mathrm{~mL}$ was

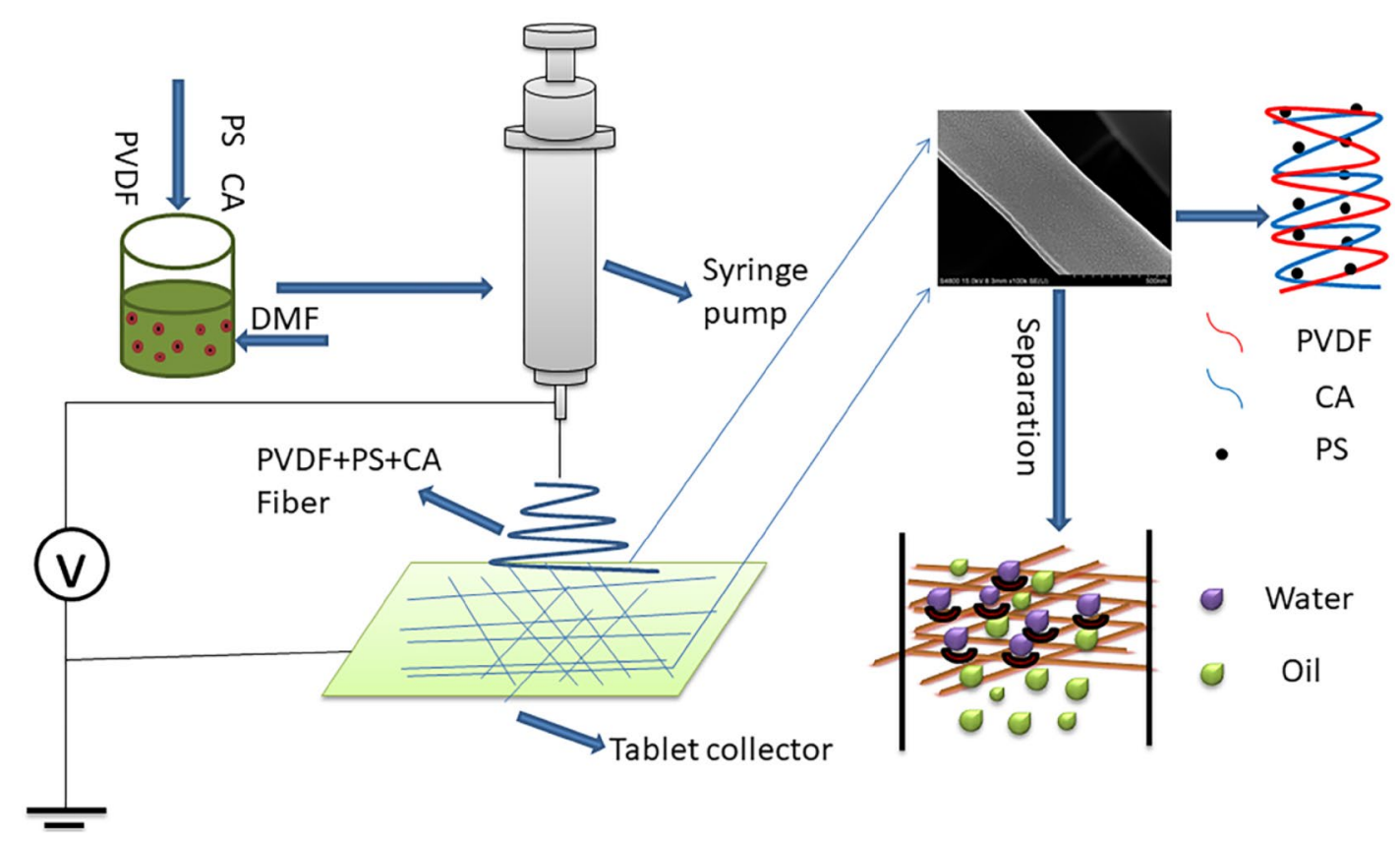

Fig. 1 Schematic illustration of electrospinning and oil-water separation

Table 1 Membrane information

\begin{tabular}{llllllllrr}
\hline Membrane & M-0-3/7 & M-0-5/5 & M-0-7/3 & M-2.5-3/7 & M-5-3/7 & M-7.5-3/7 & M-10-3/7 & M-12.5-3/7 & M-15-0/0 \\
\hline PVDF (\%) & 0 & 0 & 0 & 2.5 & 5 & 7.50 & 10 & 12.50 \\
PS (\%) & 4.50 & 7.50 & 10.50 & 3.75 & 3 & 2.25 & 1.50 & 0.75 \\
CA (\%) & 10.50 & 7.50 & 4.50 & 8.75 & 7 & 5.25 & 3.50 & 1.75 & 0 \\
\hline
\end{tabular}


used as a replenishing solution. Stir oil-water mixture with a glass rod as evenly as possible while poured into the separator. First of all, quickly pour $100 \mathrm{~mL}$ of the mixture and then continue to replenish the mixture as the oil was separated so that the liquid level above the separator is kept at the same height, until all the mixture in the beaker was poured into the separator. The separation time from the beginning to the end is recorded. The oil flux is calculated by the following formula [21]:

$F=\frac{Q}{A \cdot t}$

Here, $F$ is defined as the oil flux $\left(\mathrm{L} \mathrm{m}^{-2} \mathrm{~h}^{-1}\right) ; Q$ is the total volume (L) of the filtrate, $A$ is the effective area of the membrane $\left(\mathrm{m}^{2}\right)$; and $t$ is the time (h) from the start to the end of the separation.

After the separation was completed, the filtrate was harvested and weighed. Calculate the oil-water separation efficiency applying the following formula [26]:

$E=\frac{M_{1}}{M_{0}} \times 100 \%$

where $E$ represents the oil-water separation efficiency (\%), $M_{0}$ is the mass $(\mathrm{g})$ of the oil before separation, and $M_{1}$ is the mass ( $\mathrm{g}$ ) of the filtrate after separation.

Then, the filtrate was sequentially distilled, and the mass of the residual water was promptly weighed. The water rejection $R(\%)$ was normalized using the following formula [10]:

$R=\left(1-\frac{C_{p}}{C_{f}}\right) \times 100 \%$

$C_{f}$ is the concentration of water in the mixture before separation, and $C_{p}$ is the concentration of water in the filtrate. All test data is summarized in Table 2.

\subsection{Characterization}

The microstructure and fiber diameter of nine different nanofiber membranes were observed via a scanning electron microscope (Hitachi S4800), and the fiber morphology and the particle diameter attached to the fiber surface were obtained from the SEM image; The interface parameter integrated test system (KRVSS.DSA30S) was utilized to analyze the contact angle of the films. The pore size, pore volume and specific surface area of the membranes were examined by Brunauer-Emmett-Teller (Quantachrome iQ) on the result of nitrogen multilayer adsorption. BET curves are provided in support materials (Figures S1-S9). The computer controlled electronic universal testing machine (WDW-1000) was tested for stress-strain curves to characterize the mechanical properties of the samples. The film materials were cut into a length of $10 \mathrm{~cm}$, a width of $1 \mathrm{~cm}$ and a thickness of $0.1 \mathrm{~mm}$ rectangular strips clamped on the fixture for testing. The elemental composition of the fiber surface is analyzed by X-ray photoelectron spectrometer (XPS, Escalab 250Xi).

\section{Results and discussion}

\subsection{Surface morphology}

Electrospinning is deemed as a facile method to fabricate porous-structured fibers with high surface area, uneven pore size and ultrafine fibers. The morphology and structure of the fibers were focused on the pore diameter which is intimately related to the oil flux of the membrane, the fiber diameter and the roughness of the fiber surface directly influencing the wettability of the membrane by SEM. It is obvious that the diameter of Fig. $2 i$ is finer than other films. This may be due to the fact that the composite fiber has a higher interaction between the macromolecular

Table 2 Membrane performance comparison

\begin{tabular}{|c|c|c|c|c|c|c|c|c|c|}
\hline Membrane & $\begin{array}{l}\text { Pore } \\
\text { diameter } \\
(\mathrm{nm})\end{array}$ & $\begin{array}{l}\text { Pore } \\
\text { volume } \\
\left(\mathrm{cm}^{2} / \mathrm{g}\right)\end{array}$ & $\begin{array}{l}\text { Surface } \\
\text { area } \\
\left(\mathrm{m}^{2} / \mathrm{g}\right)\end{array}$ & Pressure $(\mathrm{kPa})$ & $\begin{array}{l}\text { Oil adsorp- } \\
\text { tion capac- } \\
\text { ity }\end{array}$ & $\begin{array}{l}\text { Water } \\
\text { contact } \\
\text { angle }\end{array}$ & Rejection (\%) & $\begin{array}{l}\text { Separation } \\
\text { efficiency } \\
(\%)\end{array}$ & $\begin{array}{l}\text { Separation } \\
\text { efficiency } \\
\left(\mathrm{L} \mathrm{m}^{-2} \mathrm{~h}^{-1}\right)\end{array}$ \\
\hline$M-0-3 / 7$ & 1.618 & 0.088 & 27.620 & 0.73 & 16.42 & 128.64 & & & \\
\hline$M-0-5 / 5$ & 1.618 & 0.059 & 16.890 & 0.73 & 7.71 & 121.48 & & & \\
\hline$M-0-7 / 3$ & 1.616 & 0.035 & 8.660 & 0.73 & 6.42 & 122.86 & & & \\
\hline$M-2.5-3 / 7$ & 1.615 & 0.028 & 16.186 & 0.73 & 14.28 & 124.29 & 98.8 & 98.5 & 942.36 \\
\hline$M-5-3 / 7$ & 1.617 & 0.025 & 16.816 & 0.73 & 13.12 & 126.44 & 99.2 & 99.1 & 1256.47 \\
\hline$M-7.5-3 / 7$ & 1.767 & 0.048 & 27.355 & 0.73 & 13.67 & 129.97 & 98.9 & 98.8 & 1404.31 \\
\hline$M-10-3 / 7$ & 2.148 & 0.135 & 78.321 & 0.73 & 32.4 & 133.05 & 99.5 & 99.4 & 1935.67 \\
\hline$M-12.5-3 / 7$ & 1.615 & 0.100 & 29.528 & 0.73 & 23.06 & 130.11 & 99.2 & 99.6 & 1302.18 \\
\hline$M-15-0 / 0$ & 1.617 & 0.018 & 7.276 & 0.73 & 9.68 & 132 & 98.4 & 98.1 & 394.6 \\
\hline
\end{tabular}




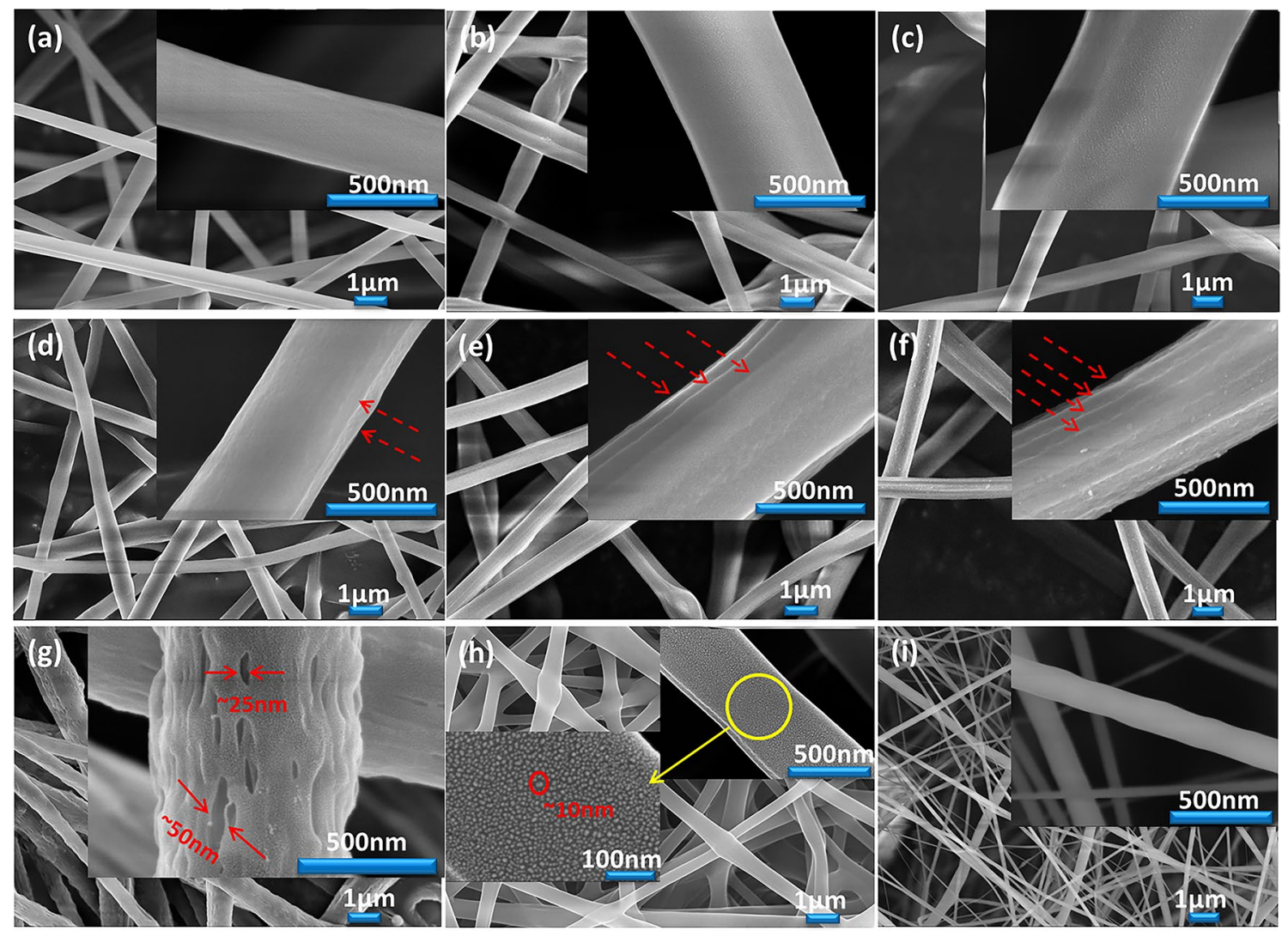

Fig. 2 SEM images of nine different ratios of membrane materials. a Membrane $\mathrm{M}-0-3 / 7$; b membrane $\mathrm{M}-0-5 / 5$; c membrane $\mathrm{M}-0$ 7/3; d membrane $M-2.5-3 / 7$ (red dotted arrow indicates the wrinkles of the fiber); e membrane M-5-3/7 (red dotted arrow indicates the wrinkles of the fiber); $\mathbf{f}$ membrane $M-7.5-3 / 7$ (red dotted arrow

chains than the single fiber, and the composite fiber is not easily pulled by the external force. Figure $2 \mathrm{a}-\mathrm{c}$ don't show significant changes under the observation of scanning electron microscopy. The Brunauer-Emmett-Teller test found that the pore size and specific surface area were different. As indicated by the red arrow in Fig. 2d, when the PVDF concentration is $2.5 \mathrm{wt} \%$, the ridgelines of the two distinct protrusions can be distinctly observed. As shown in Fig. 2e, when the PVDF concentration increases to $5 \mathrm{wt} \%$, the three distinct ridges marked by the red arrow can be noted and there are four at $7.5 \mathrm{wt} \%$ PVDF concentration in Fig. 2f. The shape of wavy wrinkle is becoming more and more palpable with the increase of PVDF components, which is a variation tendency of the fiber surface from smooth to roughness. As shown in Fig. $2 \mathrm{~g}-\mathrm{h}$, we clearly observed the surface of $\mathrm{M}-10-3 / 7$ and $\mathrm{M}-12.5-3 / 7$ covered about $10 \mathrm{~nm}$ PS particles. Due to the rapid evaporation of the solvent during electrospinning, not only the concentration of the polymer is continuously increased, but also the surface temperature of the fiber is rapidly lowered. The combination of the increase in concentration indicates the wrinkles of the fiber); $\mathbf{g}$ membrane $\mathrm{M}-10-3 / 7$ (red solid arrow indicates the pore diameter of the fiber); $\mathbf{h}$ membrane $\mathrm{M}-12.5-3 / 7$ (red circle indicates PS nanospheres and yellow circle is the enlarged area); i membrane M-15-3/7

and the decrease in temperature causes the polymer solution to cross the two-node line from the steady state to the unsteady state to cause phase separation. A plausible formation process of fibers is as follows. There are no chemical bonds between the three polymers and same macromolecule tends to form a solid phase. PS is easy to cluster together and forming nanoparticle structure. The solvent DMF phase and other polymer phases were separated. As further solvent volatilization, PS nanoparticle appears on the surface of the fiber. When the concentration of PVDF increased to $10 \mathrm{wt} \%$, membrane $\mathrm{M}-10-3 / 7$ exhibited a maximum roughness and even pores size ranging from $25 \mathrm{~nm}$ to $50 \mathrm{~nm}$ in Fig. 2g. At $12.5 \mathrm{wt} \%$ PVDF concentration, the wrinkles of the fiber are significantly reduced, but it can still be seen that the nanospheres are evenly distributed on the surface of the fiber as marked in Fig. $2 \mathrm{~h}$.

As shown in Fig. 3, in order to determine that the main component of the nano-sized particles on the surface of the fiber is PS, we prepared a PVDF-CA blend membrane under the same spinning conditions as a control. The surface of the control sample fiber is smooth, whereas the 
Fig. 3 SEM images of $\mathrm{M}-10-3 / 7$ and control sample (absence of PS at the same concentration

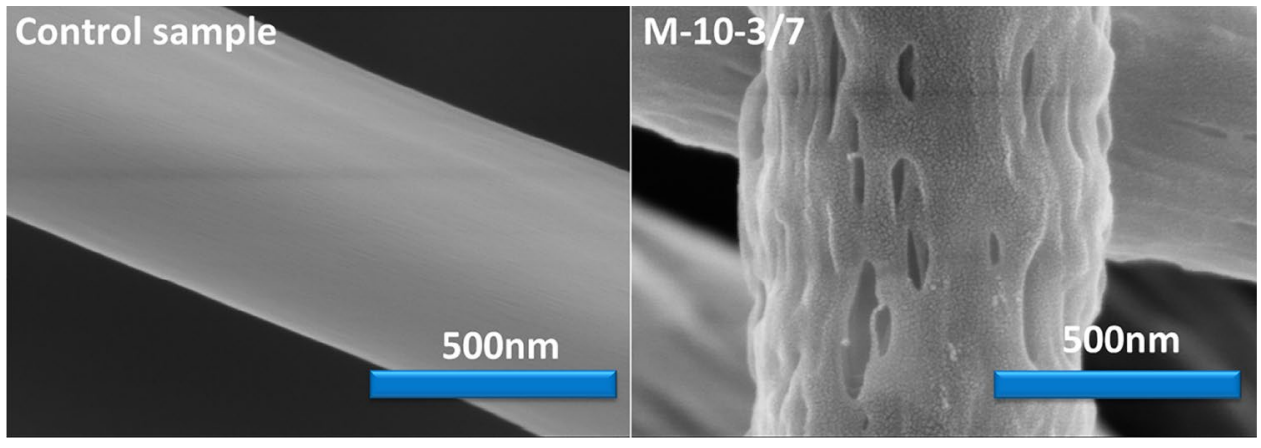

surface of $M-10-3 / 7$ exists a rough structure formed by dense nanoparticles. In addition, XPS of M-12.5-3/7 and M-15-0/0 were tested to analyze the elemental composition of the fiber surface (Figure S10 and Table S1).

\subsection{Wettability of membranes}

A high selective wettability to oil over water is a crucial factor for oil-water separation membranes [41]. Herein, the wettability of all films was characterized by static contact angle. An oil contact angle of near $0^{\circ}$ was observed for all the membranes (Fig. 4a), where oil droplets can penetrate into the membrane instantaneously. A maximum magnitude of hydrophobicity was achieved for membrane M-0-3/7 (Fig. 4b). This is attributed to the combined effects of fiber's microstructure and the polymer component. CA is well recognized as a hydrophilic polymer. Theoretically, expansion of CA concentration increases the number of hydrophilic macromolecules that make up the fiber, but in the context of electrospinning, roughness of fiber surface plays a crucial role in the hydrophobicity of the membrane [42]. PVDF is embroiled in fiber formation as shown in Fig. 4c, as the PVDF concentration ranged from 2.5 to $10 \mathrm{wt} \%$, the static water contact angle demonstrated an upward trend. The hydrophobic nature of PVDF is the decisive factor for the increase of contact angle. When the PVDF concentration reaches $10 \mathrm{wt} \%$, the water contact angle achieved a maximum of $133^{\circ}$. The wettability mainly depends on the roughness of the fiber surface and the surface free energy. From the SEM image, we found that the roughness of $M-12.5-3 / 7$ is less than the roughness of M-10-3/7. Evidently, the roughness dominates the wettability, so the membrane of $\mathrm{M}-12.5-3 / 7$ has a small contact angle. M-15-0/0 is composed of PVDF entirely, (a)

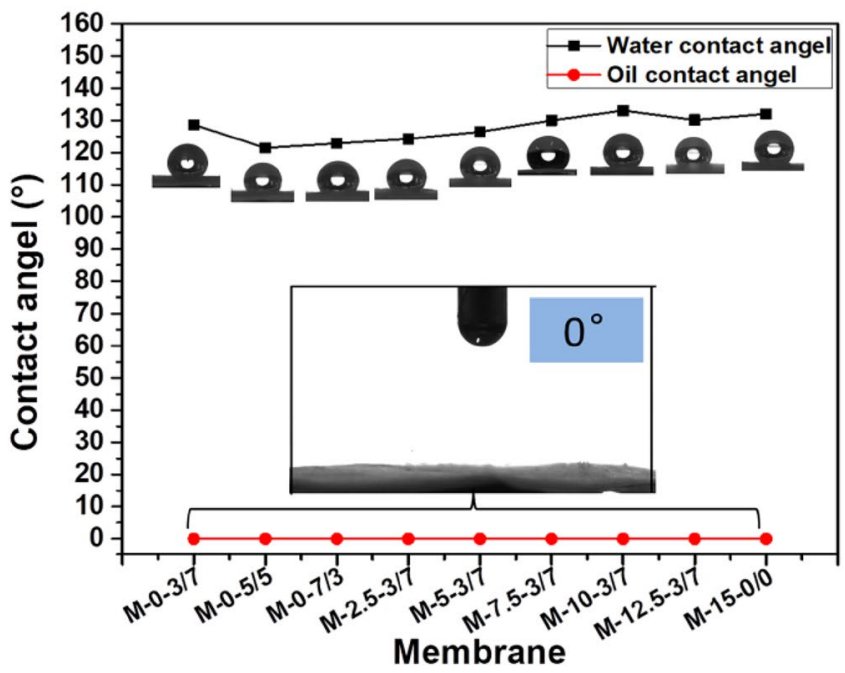

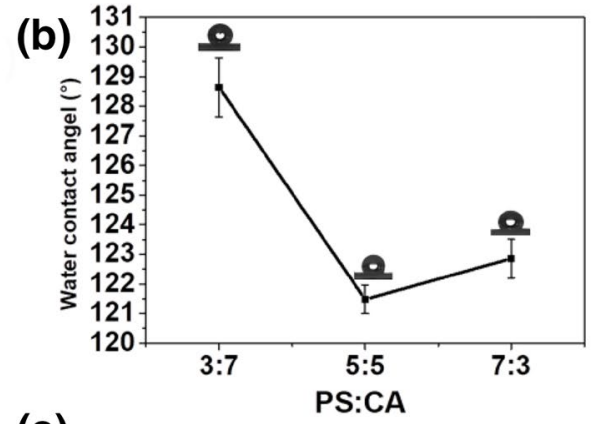

(c)

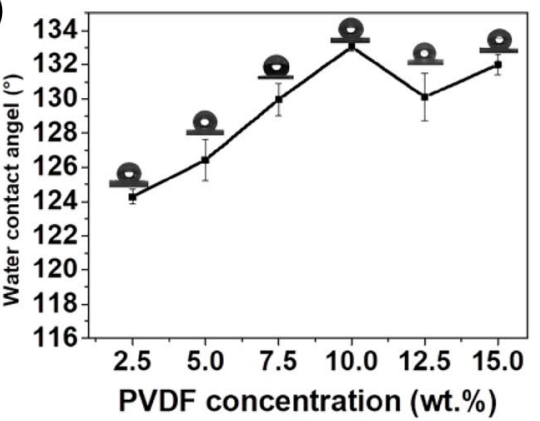

Fig. 4 a Static oil contact angle and water contact angle of nine different membrane materials, $\mathbf{b}$ three different ratios of oil contact angles for PS and CA. c Oil contact angle of membranes prepared with different PVDF concentrations 
and its composition is the main influencing factor. Due to the excellent hydrophobic properties of PVDF, the contact angle is further increased. The water contact angle of the pure PVDF film also reached $133^{\circ}$, which is almost identical to what is reported in other literature [43].

In order to demonstrate the hydrophobic properties in a kinetic manner, $50 \mu \mathrm{L}$ of water droplet stained with methylene blue was dropped onto the membrane surface, and the spread area was observed with time. As shown in Fig. 5a-f, water droplet was changed from sphere to hemisphere and then disappeared. After $10 \mathrm{~h}$, the spread area of the residual methylene blue remaining on the membrane did not change significantly compared with pristine contact area. The water droplet did not diffuse and penetrate through the membrane, but evaporated into the air. The above phenomenon indicates that $\mathrm{M}-10-3 / 7$ has the superiority of durable hydrophobicity. The stability primarily benefits from the topography of the fiber surface, which is reflected in two aspects, including the PS microspheres anchored to the fiber surface and surface wrinkles and bulges, both of which are homologous to the nanostructure composed of the papillae and waxy substances on the surface of the lotus leaf. Barthlott and Neinhuis [44] This rough structure on the fiber surface was surrounded by air, so the gap between them can't be filled with water, that being said, fiber surface and water droplets cannot fully contact due to air barrier. This phenomenon is consistent with "Cassie-Baxter regime". [27] Fig. $5 g$ shows the reverse digital photo of Fig. $5 f$ without any trace of penetration only at a thickness of about $0.1 \mathrm{~mm}$, further demonstrating the membrane's resistance to water. Similarly, we took
$50 \mu \mathrm{L}$ of chloroform dyed by Sudan III on M-10-3/7, and the oil droplets diffused into the membrane instantaneously Fig. $5 \mathrm{~h}$, indicating that the membrane has high-oleophilic properties, which provides a prerequisite for "oil passage".

\subsection{Oil sorption capacity}

The oil absorption capacity was measured to compare the affinity to oil. As shown in Fig. 6a, membrane M-0-3/7 exhibited maximum oil absorption of $16.42 \mathrm{~g} / \mathrm{g}$. The high affinity of membrane M-0-3/7 was further verified from the spread area of an oil droplet. Typically, $50 \mu \mathrm{L}$ chloroform stained by Sudan III was dropped onto the membrane surface. The spread area is in the sequence of $\mathrm{M}-0-3 / 7>\mathrm{M}-0$ $5 / 5>\mathrm{M}-0-7 / 3$ (Fig. 7). This phenomenon is consistent with the oil absorption capacity. Hence, the optimum ratio of PS to $C A$ is 3:7. Preparation of porous CA has been intensively investigated by electrospinning, which leads to a large specific surface area. [42] As shown in Table 2, the membrane $M-0-3 / 7$ possessed the largest specific surface area of $27.620 \mathrm{~m}^{2} / \mathrm{g}$. Moreover, the pore volume also reaches the maximum, indicating a larger contact area between oil and fiber when oil penetrates into the membranes. Therefore, high content of CA would improve the porosity, specific surface area and high oil absorption simultaneously. As indicated in Fig. $6 \mathrm{~b}$, oil sorption capacity is similar for membrane $\mathrm{M}-2.5-3 / 7, \mathrm{M}-5-3 / 7$ and $\mathrm{M}-7.5-3 / 7$ and the increase of PVDF content had little effect on oil sorption capacity. However, membrane $\mathrm{M}-10-3 / 7$ gave a maximum oil sorption capacity when the PVDF content increased to $10 \mathrm{wt} \%$. This is attributed to the formation of dense pores

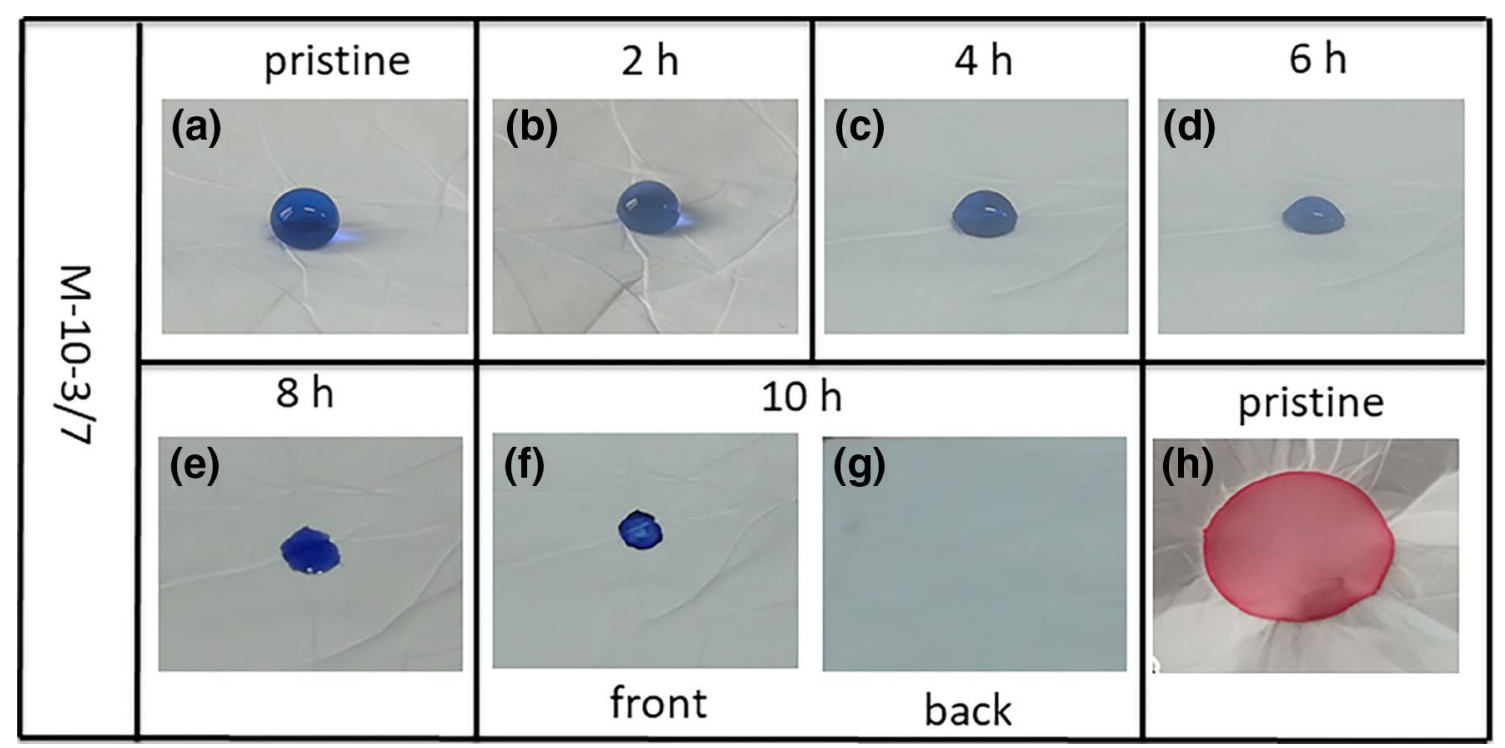

Fig. 5 a-f Digital photos of a $50 \mu \mathrm{L}$ water drop on the M-10-3/7 at different time periods; $\mathbf{g}$ the back of $\mathbf{f} ; \mathbf{h}$ digital photo of a $50 \mu \mathrm{L}$ oil drop on the $\mathrm{M}-10-3 / 7$ 
Fig. 6 a Three different ratios of oil sorption capacity for PS and CA. b Oil sorption capacity of membranes prepared with different PVDF concentrations

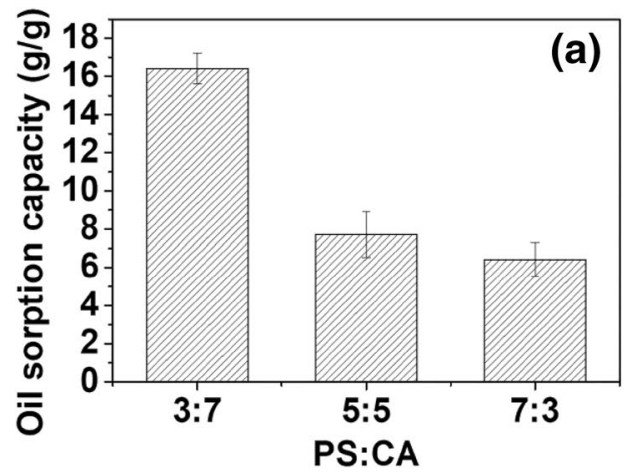

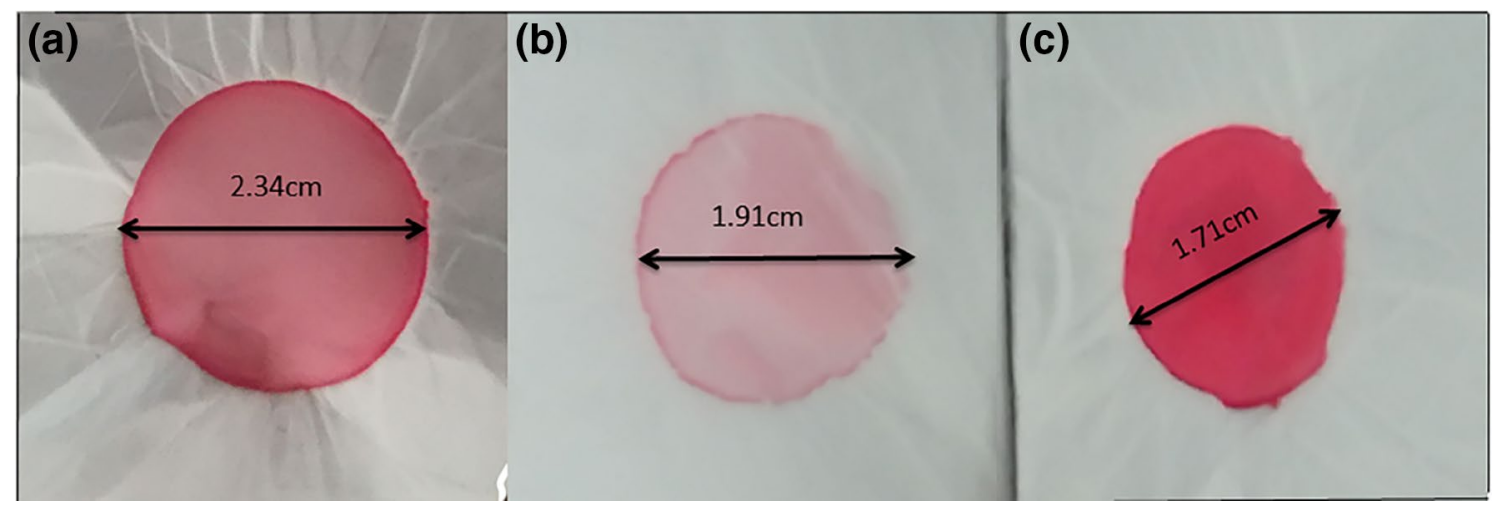

Fig. 7 Digital photos of $50 \mu \mathrm{L}$ oil droplets spread on membranes a $M-0-3 / 7, \mathbf{b} M-0-5 / 5$ and $\mathbf{c} M-0-7 / 3$, respectively

inside the fiber. In order to get more insight on the membrane texture, $\mathrm{N}_{2}$ isotherms at $77 \mathrm{~K}$ were measured to calculate the specific surface area, pore volume and pore size distribution. As shown in Table 2, membrane M-10-3/7 obtained the largest specific surface area, pore volume, and pore size, which were $78.321 \mathrm{~m}^{2} / \mathrm{g}, 0.135 \mathrm{~cm}^{2} / \mathrm{g}$ and $2.148 \mathrm{~nm}$, respectively.

\subsection{Separation performance of membranes}

Above analysis indicates that PVDF-PS-CA membranes have a great potential for oil-water separation. For further verification, oil flux and water rejection were tested. The membrane was cut into a disk-shape with diameter of $5 \mathrm{~cm}$ and fixed between the funnel and conical flask. The effective filtration area is $\sim 12.6 \mathrm{~cm}^{2}$. The thickness of the membrane was $\sim 0.1 \mathrm{~mm}$. A mixture of heavier oil (chloroform) dyed with Sudan III and water stained by methylene blue was poured into the funnel. As shown in Fig. 8a, the red oil was filtered to the flask, and the water was retained on top of the membrane. The driving force is only the gravity calculated to be $\sim 0.73 \mathrm{kPa}$. However, it should be noted that the PS-CA membranes were failed to test due to the low mechanical strength and severe swelling by oil. The oil flux of PVDF-PS-CA membranes was measured and shown in Fig. 8b. The oil flux increases gradually with PVDF content. A maximum value of $1935.67 \mathrm{~L} \mathrm{~m}^{-2} \mathrm{~h}^{-1}$ was achieved at $10 \mathrm{wt} \%$ PVDF loading. This can be explained by the increased porosity as evidenced by BET and SEM image shown above. The pure PVDF membrane showed an oil flux of $394.5 \mathrm{~L} \mathrm{~m}^{-2} \mathrm{~h}^{-1}$. Meanwhile, $>99 \%$ of the water was rejected by the PVDF-PS-CA membrane. The separation efficiency of all membranes is $>99 \%$ as shown in Fig. 8 c. The high separation efficiency makes PVDF-PS-CA membrane great potential for industrial application.

\subsection{Mechanical properties}

The mechanical strength of the membrane is another important parameter for the practical application. Herein, stress-strain test was used to evaluate the mechanical property of our membranes. From the trend of stress-strain in Fig. 9 and the data in Table 3, M-10-3/7 achieved the highest tensile strength value of $10.22 \mathrm{MPa}$ and the strain reached $39.2 \%$. The tensile strength of $\mathrm{M}-0-3 / 7$ without PVDF is only $4.78 \mathrm{MPa}$, indicating that the addition of PVDF improves the mechanical properties of the membrane material greatly; comparing the M-10-3/7 and M-15-0/0 curves, pure the PVDF membrane tensile strength reaches $10.07 \mathrm{MPa}$, which comes near to 


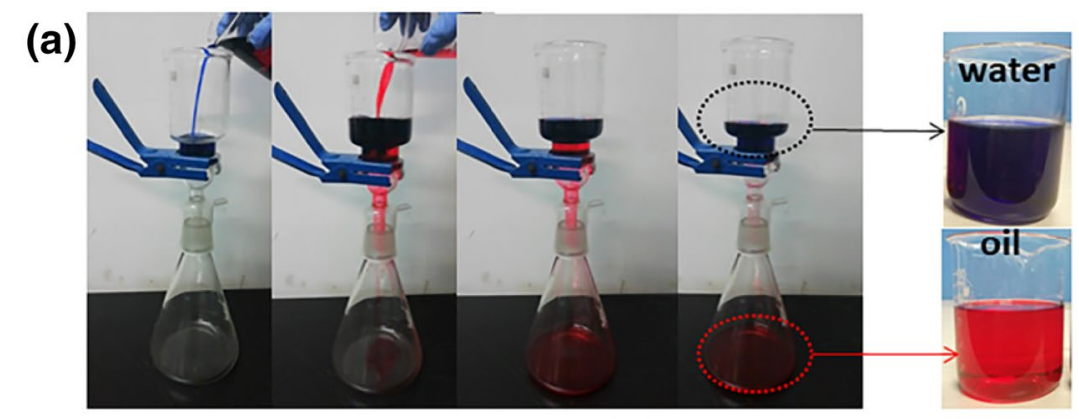

(b)



(c)

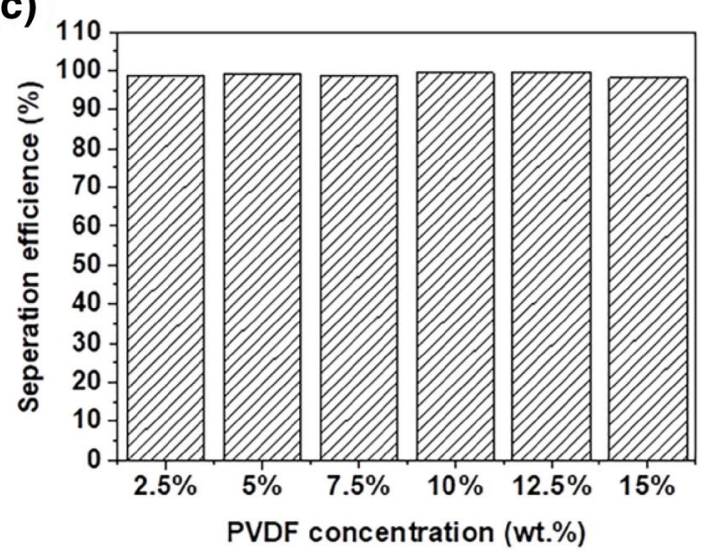

Fig. 8 a Schematic diagram of oil-water separation of M-10-3/7 with water stained by methylene blue, chloroform stained by Sudan III. b Oil flux at different PVDF concentrations in PVDF-PS-CA system. c Separation efficiency of different PVDF concentrations in PVDF-PS-CA system

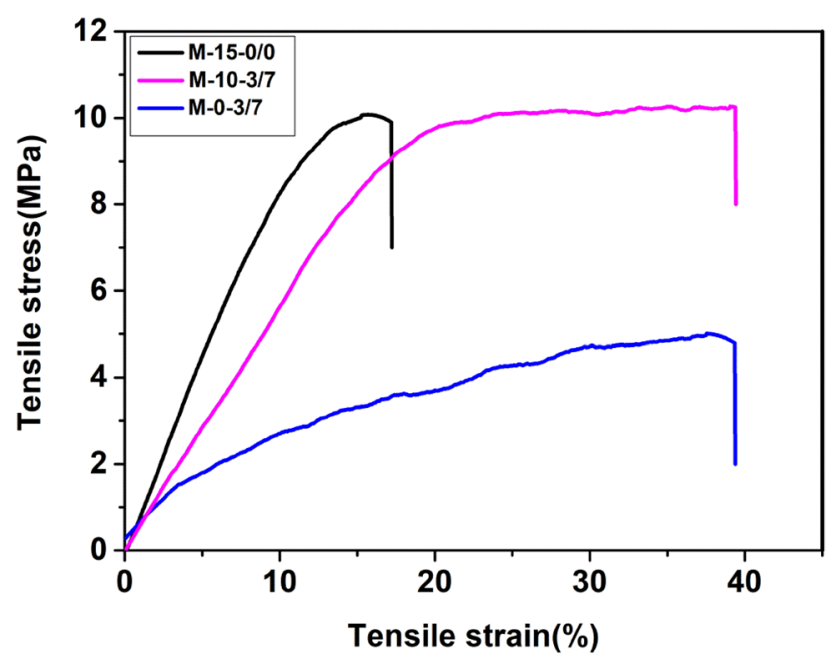

Fig. 9 Stress-strain curves of $M-15-0 / 0, M-10-3 / 7$ and $M-0-3 / 7$

M-10-3/7; However, the tensile strain is only $4.78 \%$, which is much smaller than the other two, because the addition of CA and PS increases the flexibility of the fiber. From stress-strain, oil flux, separation efficiency and testing condition, comparing our work with other literatures reported in recent years, comprehensive performance of M-10-3/7 is superior in all aspects as shown in Table 4.
Table 3 Tensile stress-tensile strain information of membranes

\begin{tabular}{lcc}
\hline Membrane & Tensile strain (\%) & $\begin{array}{l}\text { Tensile } \\
\text { stress } \\
\text { (MPa) }\end{array}$ \\
\hline M-0-3/7 & 39.35 & 4.78 \\
$M-10-3 / 7$ & 39.2 & 10.22 \\
$M-15-0 / 0$ & 4.78 & 10.07 \\
\hline
\end{tabular}

\subsection{Oil-water separation mechanism}

Figure 10 shows a plausible mechanism of oil-water separation based on our PVDF-PS-CA membranes. Once the oil-water mixture contacts the membrane surface, the oil droplets would be quickly adsorbed and diffuse across the membrane due to the synergistic effects of gravity force and high affinity. The interconnected pores between the fibers and the nanopores within the fiber serve as a "high pathway" for oil diffusion. However, the adsorption or diffusion of water would be rejected because of the highly hydrophobic property of PS nanoparticles. Water droplets are inferior due to the competitive adsorption with oil droplets on the membrane surface. Therefore, the water droplets were rejected and the oil droplets spread out as an "oil film" on the membrane surface. Three forces would 
Table 4 Comparision between recently reported membranes

\begin{tabular}{|c|c|c|c|c|c|c|c|}
\hline Membrane & Tensile strain (\%) & $\begin{array}{l}\text { Tensile stress } \\
\text { (MPa) }\end{array}$ & $\begin{array}{l}\text { Separated com- } \\
\text { pound }\end{array}$ & $\begin{array}{l}\text { Testing condition } \\
(\mathrm{kPa})\end{array}$ & $\begin{array}{l}\text { Oil flux } \\
\left(\mathrm{L} \mathrm{m}^{-2} \mathrm{~h}^{-1}\right)\end{array}$ & $\begin{array}{l}\text { Separation } \\
\text { efficiency } \\
(\%)\end{array}$ & Refs. \\
\hline $\begin{array}{l}\text { Polyacrylonitrile } \\
\text { nanofibers }\end{array}$ & Unknown & Unknown & $\begin{array}{l}\text { 1,2-dibromoe- } \\
\text { thane }\end{array}$ & Gravity-driven & 1061.1 & Efficient & {$[10]$} \\
\hline $\begin{array}{l}\text { 2D heterostruc- } \\
\text { ture }\end{array}$ & Unknown & Unknown & Oil & 10 & About 600 & A bout 99 & {$[20]$} \\
\hline $\begin{array}{l}\text { Polycaprolac- } \\
\text { tone/beeswax }\end{array}$ & 40 & 6.5 & Petrol & Gravity-driven & 1599 & 98.6 & {$[21]$} \\
\hline $\begin{array}{l}\text { Polycaprolac- } \\
\text { tone/beeswax }\end{array}$ & 40 & 6.5 & Diesel & Gravity-driven & 1066 & 98.8 & {$[21]$} \\
\hline $\begin{array}{l}\text { Polycaprolac- } \\
\text { tone/beeswax }\end{array}$ & 40 & 6.5 & Kerosene & Gravity-driven & 1492 & 97.9 & {$[21]$} \\
\hline $\begin{array}{l}\text { Carbon-silica } \\
\text { composite } \\
\text { nanofibrous }\end{array}$ & Unknown & Unknown & Isooctane & Gravity-driven & $1719.1 \pm 36.2$ & Efficient & {$[23]$} \\
\hline $\begin{array}{l}\text { Hierarchical } \mathrm{TiO}_{2} \\
\text { nanotubes }\end{array}$ & Unknown & Unknown & Petroleum & Gravity-driven & $311-1357$ & $97.2-99.4$ & [9] \\
\hline $\begin{array}{l}\text { Polyurethane/ } \\
\text { silica composite }\end{array}$ & 26 & 0.78 & Different oils & 5 & $428-800$ & Above 99.0 & {$[45]$} \\
\hline $\begin{array}{l}\text { This work (M-10- } \\
3 / 7 \text { ) }\end{array}$ & 39.2 & 10.22 & Chloroform & $\begin{array}{c}\text { Gravity-driven } \\
\text { (about } 0.73 \text { ) }\end{array}$ & 1935.67 & 99.4 & This study \\
\hline
\end{tabular}

Fig. 10 Schematic diagram illustrating the plausible oilwater separation mechanism

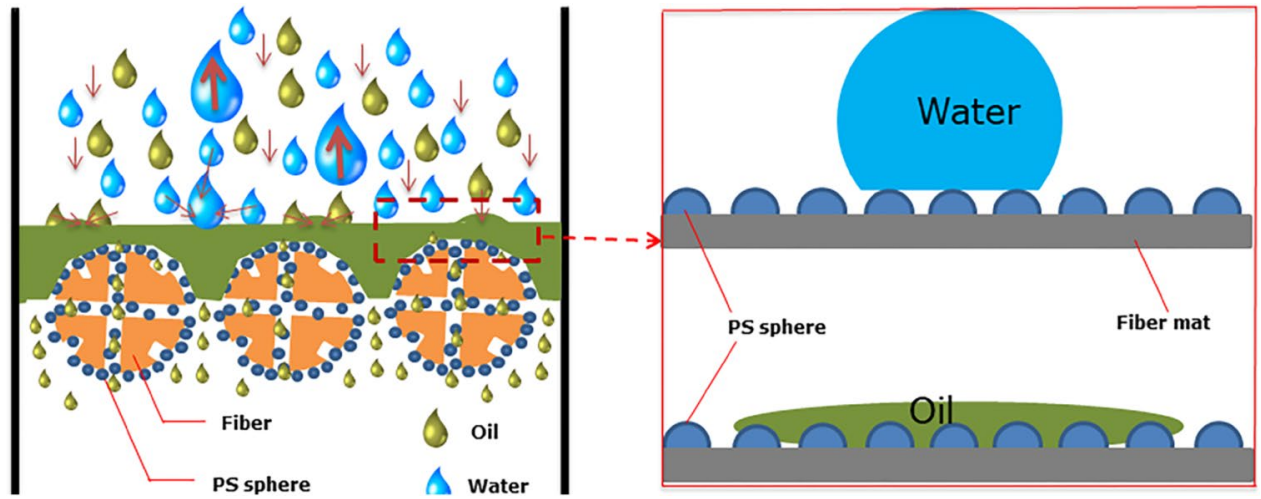

be applied on the water droplets, including upward buoyancy, oil film repulsive force and downward gravity. The combined force of buoyancy and repulsive force is bigger than gravity. In the end, the small water droplets aggregate together to form big water droplets and keep in the retentate side.

\section{Conclusions}

PVDF-PS-CA membranes were successfully prepared by electrospinning for oil-water separation. The mechanical strength of the membranes was significantly enhanced by blending PVDF, giving a maximum tensile stress of $10.22 \mathrm{MPa}$; the texture of the fibers was well manipulated by $C A$, enduring a high oil flux; superior hydrophobic property was achieved by forming $10 \mathrm{~nm}$ sized PS nanospheres on the fibers' surface. The composition of the membrane was optimized to achieve efficient oil/water separation. Typically, the membrane $\mathrm{M}-10-3 / 7$ consisting of $10 \mathrm{wt} \%$ PVDF, $1.5 \mathrm{wt} \% \mathrm{PS}$ and $3.5 \mathrm{wt} \%$ CA exhibited an oil flux as high as $1935.67 \mathrm{~L} \mathrm{~m}^{-2} \mathrm{~h}^{-1}$ only driven by the gravity force of $0.73 \mathrm{kPa}$ and an oil-water separation efficiency of $99.4 \%$. The high mechanical strength together with the high separation performance would pave the way of PVDF-PS-CA membranes for industrial oil/water separation.

Acknowledgements This work was supported by the National Natural Science Foundation of China (No. 21573174). 


\section{Compliance with ethical standards}

Conflict of interest The authors declare that they have no conflict of interest.

\section{References}

1. Lo YH, Yang CY, Chang HK, Hung WC, Chen PY (2017) Bioinspired diatomite membrane with selective superwettability for oil/ water separation. Sci Rep 7:1426

2. Lee MW, An S, Latthe SS, Lee C, Hong S, Yoon SS (2013) Electrospun polystyrene nanofiber membrane with superhydrophobicity and superoleophilicity for selective separation of water and low viscous oil. ACS Appl Mater Interfaces 5:10597-10604

3. Tran VT, Lee BK (2017) Novel fabrication of a robust superhydrophobic PU@ZnO@ $\mathrm{Fe}_{3} \mathrm{O}_{4} @ S A$ sponge and its application in oil-water separations. Sci Rep 7:17520

4. Wang B, Liang W, Guo Z, Liu W (2015) Biomimetic super-lyophobic and super-lyophilic materials applied for oil/water separation: a new strategy beyond nature. Chem Soc Rev 44:336-361

5. Ge J, Zhang J, Wang F, Li Z, Yu J, Ding B (2017) Superhydrophilic and underwater superoleophobic nanofibrous membrane with hierarchical structured skin for effective oil-in-water emulsion separation. J Mater Chem A 5:497-502

6. Gondal MA, Sadullah MS, Qahtan TF, Dastageer MA, Baig U, McKinley GH (2017) Fabrication and wettability study of $\mathrm{WO}_{3}$ coated photocatalytic membrane for oil-water separation: $A$ comparative study with $\mathrm{ZnO}$ coated membrane. Sci Rep 7:1686

7. Bae J, Kim H, Kim KS, Choi H (2018) Effect of asymmetric wettability in nanofiber membrane by electrospinning technique on separation of oil/water emulsion. Chemosphere 204:235-242

8. Meng $X$, Wang $M$, Heng L, Jiang L (2018) Underwater mechanically robust oil-repellent materials: combining conflicting properties using a heterostructure. Adv Mater 30:1706634

9. Li L, Liu Z, Zhang Q, Meng C, Zhang T, Zhai J (2015) Underwater superoleophobic porous membrane based on hierarchical $\mathrm{TiO}_{2}$ nanotubes: multifunctional integration of oil-water separation, flow-through photocatalysis and self-cleaning. J Mater Chem A 3:1279-1286

10. Li X, Wang M, Wang C, Cheng C, Wang X (2014) Facile immobilization of ag nanocluster on nanofibrous membrane for oil/water separation. ACS Appl Mater Interfaces 6:15272-15282

11. Liu H, Raza A, Aili A, Lu J, AlGhaferi A, Zhang T (2016) Sunlightsensitive anti-fouling nanostructured $\mathrm{TiO}_{2}$ coated $\mathrm{Cu}$ meshes for ultrafast oily water treatment. Sci Rep 6:25414

12. Saththasivam J, Yiming W, Wang K, Jin J, Liu Z (2018) A novel architecture for carbon nanotube membranes towards fast and efficient oil/water separation. Sci Rep 8:7418

13. Wang CF, Huang HC, Chen LT (2015) Protonated melamine sponge for effective oil/water separation. Sci Rep 5:14294

14. Ba H, Liu Y, Wang W, Duong-Viet C, Papaefthimiou V, NguyenDinh L, Tuci G, Giambastiani G, Pham-Huu C (2018) Carbon felt monoliths coated with a highly hydrophobic mesoporous carbon phase for the continuous oil sorption/filtration from water. Adv Sustain Syst 2:1800040

15. Lin J, Cai Y, Wang X, Ding B, Yu J, Wang M (2011) Fabrication of biomimetic superhydrophobic surfaces inspired by lotus leaf and silver ragwort leaf. Nanoscale 3:1258-1262

16. Haase MF, Jeon H, Hough N, Kim JH, Stebe KJ, Lee D (2017) Multifunctional nanocomposite hollow fiber membranes by solvent transfer induced phase separation. Nat Commun 8:7

17. Yue X, Zhang T, Yang D, Qiu F, Li Z, Zhu Y, Yu H (2018) Oil removal from oily water by a low-cost and durable flexible membrane made of layered double hydroxide nanosheet on cellulose support. J Clean Prod 180:307-315

18. Wang K, Han DS, Yiming W, Ahzi S, Abdel-Wahab A, Liu Z (2017) A windable and stretchable three-dimensional allinorganic membrane for efficient oil/water separation. Sci Rep 7:16081

19. Xiong Z, Lin H, Liu F, Xiao P, Wu Z, Li T, Li D (2017) Flexible PVDF membranes with exceptional robust superwetting surface for continuous separation of oil/water emulsions. Sci Rep 7:14099

20. Liu Y, Su Y, Guan J, Cao J, Zhang R, He M, Gao K, Zhou L, Jiang $Z$ (2018) 2D heterostructure membranes with sunlight-driven self-cleaning ability for highly efficient oil-water separation. Adv Func Mater 28:1706545

21. Reshmi CR, Sundaran SP, Juraji A, Athiyanathil S (2017) Fabrication of superhydrophobic polycaprolactone/beeswax electrospun membranes for high-efficiency oil/water separation. RSC Adv 7:2092-2102

22. Zou CY, Wang ZM, Ren XM, Li RR, Zhang SP, Sheng BY (2018) Application of a water stable porous metal-organic framework for 5-Fu delivery and inhibiting human spinal cord tumor cells. Inorg Chem Commun 91:91-94

23. Tai MH, Gao P, Tan BY, Sun DD, Leckie JO (2014) Highly efficient and flexible electrospun carbon-silica nanofibrous membrane for ultrafast gravity-driven oil-water separation. ACS Appl Mater Interfaces 6:9393-9401

24. Arslan O, Aytac Z, UyarT (2016) Superhydrophobic, hybrid, electrospun cellulose acetate nanofibrous mats for oil/water separation by tailored surface modification. ACS Appl Mater Interfaces 8:19747-19754

25. Islam MS, McCutcheon JR, Rahaman MS (2017) A high flux polyvinyl acetate-coated electrospun nylon $6 / \mathrm{SiO}_{2}$ composite microfiltration membrane for the separation of oil-in-water emulsion with improved antifouling performance. J Membrane Sci 537:297-309

26. Joo M, Shin J, Kim J, You JB, Yoo Y, Kwak MJ, Oh MS, Im SG (2017) One-step synthesis of cross-linked ionic polymer thin films in vapor phase and its application to an oil/water separation membrane. J Am Chem Soc 139:2329-2337

27. Chu Z, Feng Y, Seeger S (2015) Oil/water separation with selective superantiwetting/superwetting surface materials. Angew Chem 54:2328-2338

28. Lin J, Ding B, Yu J, Hsieh Y (2010) Direct fabrication of highly nanoporous polystyrene fibers via electrospinning. ACS Appl Mater Interfaces 2:521-528

29. Ding B, Lin J, Wang X, Yu J, Yang J, Cai Y (2011) Investigation of silica nanoparticle distribution in nanoporous polystyrene fibers. Soft Mater 7:8376-8383

30. Hou L, Wang L, Wang N, Guo F, Liu J, Chen Y, Liu J, Zhao Y, Jiang $L$ (2016) Separation of organic liquid mixture by flexible nanofibrous membranes with precisely tunable wettability. NPG Asia Mater 8:e334

31. Xiong Z, Liu F, Gao A, Lin H, Yu X, Wang Y, Wang Y (2016) Investigation of the heat resistance, wettability and hemocompatibility of a polylactide membrane via surface crosslinking induced crystallization. RSC Adv 6:20492-20499

32. Liu L, Yuan W (2018) A hierarchical functionalized biodegradable PLA electrospun nanofibrous membrane with superhydrophobicity and antibacterial properties for oil/water separation. New J Chem 42:17615-17624

33. Ahmed FE, Lalia BS, Hilal N, Hashaikeh R (2014) Underwater superoleophobic cellulose/electrospun PVDF-HFP membranes for efficient oil/water separation. Desalination 344:48-54

34. Obaid M, Tolba GMK, Motlak M, Fadali OA, Khalil KA, Almajid AA, Kim B, Barakat NAM (2015) Effective polysulfone-amorphous $\mathrm{SiO}_{2} \mathrm{NPs}$ electrospun nanofiber membrane for high flux oil/ water separation. Chem Eng J 279:631-638 
35. Wang X, Yu J, Sun G, Ding B (2016) Electrospun nanofibrous materials: a versatile medium for effective oil/water separation. Mater Today 19:403-414

36. Ma Q, Cheng H, Fane AG, Wang R, Zhang H (2017) Recent development of advanced materials with special wettability for selective oil/water separation. J Membrane Sci 12:2186-2202

37. Liu YQ, Zhang YL, Fu XY, Sun HB (2015) Bioinspired underwater superoleophobic membrane based on a graphene oxide coated wire mesh for efficient oil/water separation. ACS Appl Mater Interfaces 7:20930-20936

38. Yang X, He Y, Zeng G, Chen X, Shi H, Qing D, Li F, Chen Q (2017) Bio-inspired method for preparation of multiwall carbon nanotubes decorated superhydrophilic poly(vinylidene fluoride) membrane for oil/water emulsion separation. Chem Eng J 321:245-256

39. Zhang W, Shi Z, Zhang F, Liu X, Jin J, Jiang L (2013) Superhydrophobic and superoleophilic PVDF membranes for effective separation of water-in-oil emulsions with high flux. Adv Mater 25:2071-2076

40. Jiang Z, Tijing LD, Amarjargal A, Park CH, An K-J, Shon HK, Kim CS (2015) Removal of oil from water using magnetic bicomponent composite nanofibers fabricated by electrospinning. Composites Part B-Engineering 77:311-318

41. Ge JL, Zong DD, Jin Q, Yu JY, Ding B (2018) Biomimetic and superwettable nanofibrous skins for highly efficient separation of oil-in-water emulsions. Adv Funct Mater 28:10
42. Yousefi B, Gharehaghaji AA, Asgharian Jeddi AA, Karimi M (2018) The combined effect of wrinkles and noncircular shape of fibers on wetting behavior of electrospun cellulose acetate membranes. J Polym Sci, Part B: Polym Phys 56:1012-1020

43. Qing W, Shi X, Zhang W, Wang J, Wu Y, Wang P, Tang CY (2018) Solvent-thermal induced roughening: a novel and versatile method to prepare superhydrophobic membranes. J Membr Sci 564:465-472

44. Barthlott W, Neinhuis C (1997) Purity of the sacred lotus, or escape from contamination in biological surfaces. Planta 202:1-8

45. Fang W, Liu L, Guo G (2017) Tunable wettability of electrospun polyurethane/silica composite membranes for effective separation of water-in-oil and oil-in-water emulsions. Chemistry 23:11253-11260

Publisher's Note Springer Nature remains neutral with regard to jurisdictional claims in published maps and institutional affiliations. 